\title{
Seasonality of tuberculosis: the reverse of other respiratory diseases in the UK
}

\author{
A S Douglas, D P Strachan, J D Maxwell
}

Department of

Medicine and

Therapeutics,

University of

Aberdeen,

Foresterhill, Aberdeen

AB9 2ZD, UK

A S Douglas

Department of Public Health Sciences

D P Strachan

\section{Department of \\ Medicine \\ J D Maxwell}

St George's Hospital Medical School, London SW17 ORE, UK

Correspondence to: Professor A S Douglas.

Received 21 September 1995 Returned to authors 15 December 1995 Revised version received 1 February 1996 Accepted for publication 18 April 1996

\begin{abstract}
Background - In Western societies there is a winter peak in mortality, largely accounted for by respiratory and cardiovascular deaths. In view of the known seasonal variation in vitamin $D$, and of the postulated link between tuberculosis and vitamin $D$ deficiency, a study was undertaken to examine whether the presentation of tuberculosis had the same seasonal rhythm as other pulmonary infections.

Methods - Using cosinor analysis the presence or absence of seasonality was determined for 57313 tuberculosis notifications for England and Wales. OPCS data in four weekly notifications over a 10 year period (1983-92) were examined as two quinquential sets (1983-7 and 198892). These were compared with two groups of acute respiratory illness: 138992 notifications to OPCS of pneumonia deaths for 1988-92 and all admissions to Scottish hospitals with respiratory disease (252 163 cases) during 1980-4.
\end{abstract}

Results - Analysis of notifications of tuberculosis revealed a summer peak with an amplitude of $10 \%$. This pattern differs markedly from other respiratory disorders in which a winter peak and summer trough is observed.

Conclusions - The unusual seasonality of tuberculosis is currently unexplained. One possibility is that low post-winter trough levels of vitamin $D$ (which are known to affect macrophage function and cell mediated immunity) might result in impaired cellular immunity leading, after a latent period, to reactivation of dormant mycobacterial infection.

(Thorax 1996;51:944-946)

Keywords: tuberculosis, season, seasonality.

In Western societies there is a winter peak in total mortality, this rhythm being much influenced by cardiovascular and respiratory deaths which account for $60 \%$ of the total. ${ }^{1} \mathrm{~A}$ similar seasonal pattern is also seen in morbidity as measured by emergency hospital admissions for cardiovascular and respiratory disorders, with a winter peak sometimes of a very high amplitude. ${ }^{2}$ Seasonal peaks occur six months later in the Southern hemisphere, during the antipodean winter, confirming an environmental influence on these rhythms. Since most tuberculosis in the UK is pulmonary, this study examined whether presentation of tuber- culosis had the same seasonal rhythm as other pulmonary infections.

\section{Methods}

TUBERCULOSIS

Tuberculosis is a statutorily notifiable disease in the UK and these data are published in four weekly sets in the annual OPCS publication Communicable Disease Statistics from the Communicable Disease Surveillance Centre of the Public Health Service. The data (57313 notifications) for a 10 year period (1983-92) were examined as two quinquential sets, $1983-7$ and $1988-92 ; 78 \%$ of the cases of tuberculosis were pulmonary.

\section{OTHER RESPIRATORY DISEASES}

For comparison with acute respiratory illness, data from two other groups were examined: (1) deaths from pneumonia (ICD 480-486) for 1988-92 (138992 notifications) obtained in the same format from OPCS statistics with cases secondary to influenza being excluded; and (2) all 252163 admissions to Scottish hospitals (1980-4) with respiratory disease (ICD 460-519) obtained from the Information Statistics Division, Scottish Health Services in calendar months and adjusted for the number of days in each month.

ANALYSIS OF DATA

The statistical method used to determine the presence or absence of seasonality and the statistical significance of this variation was cosinor analysis. ${ }^{1}$ This fits a sinusoidal curve. The monthly regression analysis gives the multiple correlation coefficient $(\mathrm{R})$, its statistical significance ( $\mathrm{p}$ ), and the angular position in the year where the fitted sinusoidal regression line has its highest value. The extent of the seasonal fluctuation (amplitude, A) is measured as the percentage above the mean for the month of highest value (acrophase or peak).

\section{Results}

Over 10 years tuberculosis notifications showed a significant seasonality, having a summer peak with an amplitude of approximately $10 \%$ (fig 1). Notifications for "pneumonia" deaths, on the other hand, had a winter peak with an amplitude which was four times greater. Admissions for all respiratory diseases had a winter peak with an amplitude of $14.3 \%$.

\section{Discussion}

In the UK and other westernised societies the major causes of morbidity and mortality are "cardiovascular" and "respiratory" diseases, both of which have winter peaks. The extent of 
A

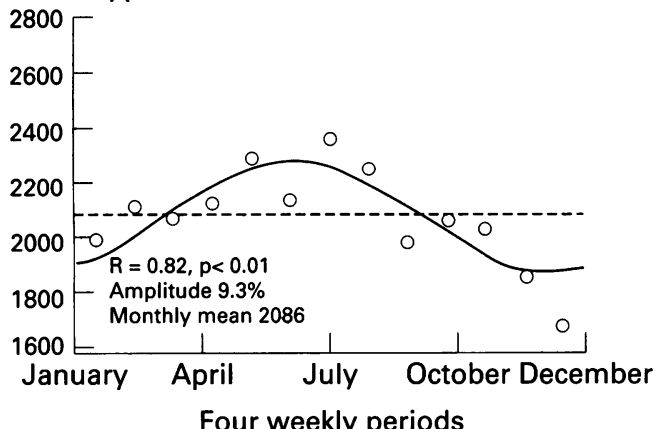

Four weekly periods

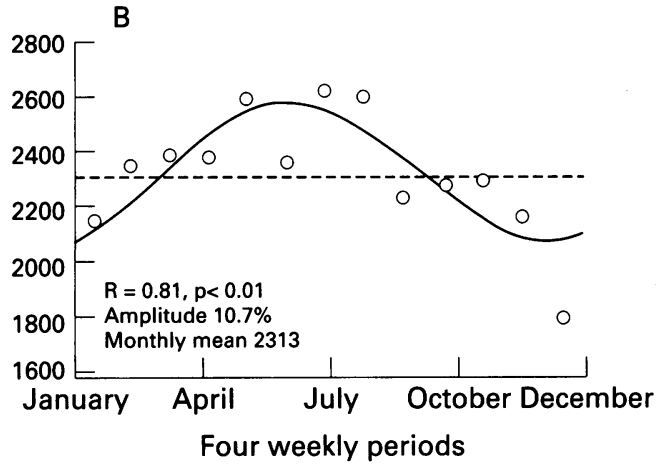

C

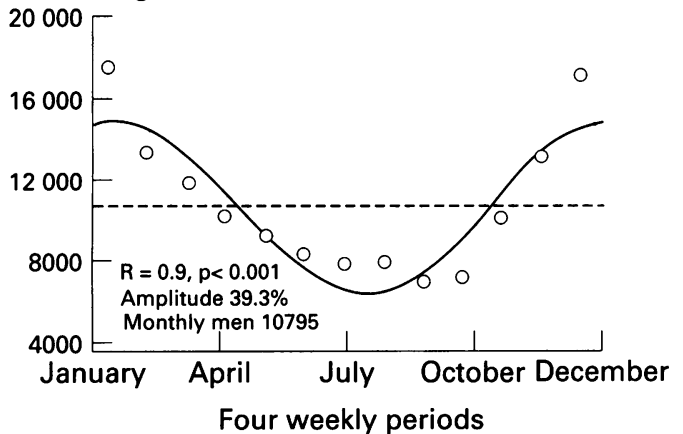

D

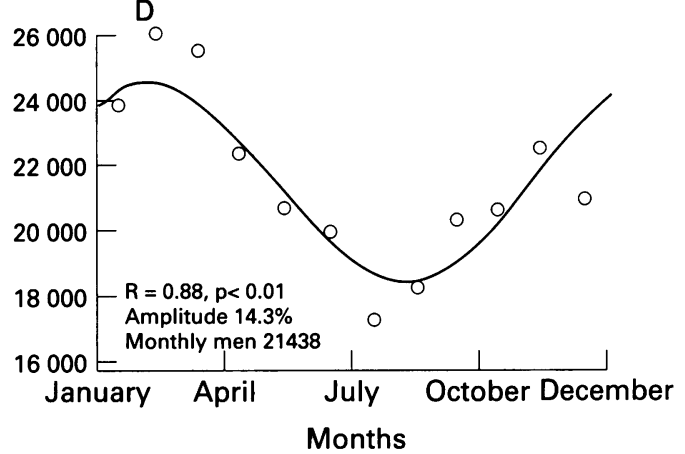

Figure 1 Results of cosinor analysis showing monthly data (expressed as a percentage of the mean monthly value) for tuberculosis reports in England and Wales for (A) 1988-92 and (B) 1983-7, (C) for pneumonia deaths in England and Wales in 1988-92, and (D) all respiratory admissions in Scotland in 1980-4. The amplitude and significance of the seasonal variation are shown in each case. All show significant seasonality, but the peak for tuberculosis occurs in fune, while for acute respiratory disorders it is in fanuary/February. The vertical axes give absolute numbers and the horizontal axes give time in four weekly intervals or calendar months.

seasonal change is greatest for "respiratory" disease (amplitude 35.7\%), and about twice that seen for "cardiovascular" diseases (amplitude $15.4 \%$ ) which, in turn, exceeds that for "all other diseases" (amplitude 13.6\%). In UK hospitals medical beds are under pressure in winter because patients stay in hospital longer and there are more cardiorespiratory admissions. $^{2}$

As yet there is no explanation for the winter seasonality of most disease and death, but with six month phase shifting the findings are the same in the Southern hemisphere so environmental factors must play a part. The specific contribution of a variety of climatic and meteorological seasonal changes including the effects of temperature, relative humidity, wind, barometric pressure, radiation spectrum of sunlight, and photoperiod is uncertain. In addition, seasonal changes in atmospheric composition or specific infections may contribute to altered respiratory pathophysiology.

In this context, our new finding that there is a summer peak for tuberculosis appears paradoxical. The data on tuberculosis represent notifications and not the date of diagnosis or onset of illness (often difficult to determine in this chronic infection). It is therefore likely that the summer peak in tuberculosis reflects disease processes which occur some months earlier. The amplitude of seasonal variation is too great to be explained by monthly variations in the number of cases detected among newly arrived immigrants. In the pre-antibiotic era, seasonal changes in the weight of patients with tuberculosis were reported, with lowest weight gains in winter and maximum weight gains in summer months. ${ }^{3}$ This suggests enhanced disease activity during the winter period.

Most cases of adult tuberculosis are thought to be due to reactivation. In most of the cases not associated with HIV infection or immunosuppressive therapy the mechanism of reactivation and development of overt tuberculosis is not well understood, but it has been ascribed to poor nutrition and socioeconomic status. ${ }^{4}$ In view of the role of cell mediated immunity in tuberculosis, it is interesting to note that there is a circannual variation in lymphocyte subsets. In healthy individuals followed over seven years there were no significant seasonal changes in the total number of white cells or total lymphocyte counts, but the absolute number of CD4+ $\mathrm{T}$ lymphocytes was lowest in summer when the CD8+ T lymphocytes were highest. ${ }^{5}$ Seasonal changes in the absolute numbers and ratios of $\mathrm{T}$ helper and suppressor cells could possibly alter cell mediated immunity, which is crucial to the host response controlling infection with Mycobacterium tuberculosis. However, the factors that regulate the seasonal changes in $T$ cell subset numbers or function remain unknown.

A link between tuberculosis and vitamin D deficiency has been postulated; indeed, sunlight exposure (phototherapy) and fish liver oils (rich in the vitamin $\mathrm{D}$ precursor ergocalciferol) were sometimes used to treat tuberculosis before the advent of antimicrobial drug treatment. ${ }^{4}$ It is now recognised that the principal source of vitamin $\mathrm{D}$ is ultraviolet radiation from sunlight, and that plasma concentrations of vitamin $\mathrm{D}$ have a striking seasonal variation with peak levels after the summer and lowest 
levels in the spring. ${ }^{7}$ A recent case-control study in south London of Asians from the Indian subcontinent (who are at greatly increased risk of tuberculosis compared with the indigenous UK population) has shown a significant trend of increasing risk of tuberculosis with decreasing frequency of meat or fish consumption. ${ }^{4}$ Vitamin D deficiency is much more common among vegetarian Asians ${ }^{8}$ and is known to have effects on immunological function in animals. ${ }^{9}$ It was suggested that vita$\min \mathrm{D}$ deficiency may be the factor in a vegetarian diet that is responsible for reduced immunocompetence with impaired cell mediated immunity and subsequent reactivation of tuberculosis in immigrant Asians. ${ }^{4}$

Taken together these findings suggest one possible explanation for the paradoxical reversed seasonality of tuberculosis in the UK. Seasonal changes in vitamin $\mathrm{D}$, with postwinter trough levels, may sufficiently impair macrophage function and cell mediated immunity $^{10}$ in some persistor status individuals to result in reactivation of dormant mycobacteria. Delays in developing clinically symptomatic disease, diagnosis, and notification could account for the summer peaks in reported incidence. This suggestion is speculative, but comparison of the seasonal patterns of tuberculosis in indigenous white subjects, vegetarian and non-vegetarian Asians in the UK would be of interest.

ASD held a Leverhulme senior emeritus research fellowship. Other financial support came from the Maryland Medical Research Institute. Dr J M Rawles developed the cosinor analysis programme.

1 Douglas AS, Al-Sayer H, Rawles, JM, Allan TM. Seasonality of disease in Kuwait. Lancet 1991;337:1393-7.

2 Douglas AS, Rawles JM, Alexander E, Allan TM. Winter pressure on hospital beds. BMF 1991;303:508-9.

3 Smith JH. Variations in the weight of the tuberculous under sanatorium treatment. $B M \mathcal{F} 1917$; 1:505-8.

4 Strachan DP, Powell KJ, Thaker A, Millard FJC, Maxwell JD. Vegetarian diet is a risk factor for tuberculosis in immigrant south London Asians. Thorax 1995;50:175-80.

5 Paglieroni TG, Holland PV. Circannual variation in lymphocyte subsets, revisited. Transfusion 1994;34:512-6.

6 Davies PDO. A possible link between vitamin D deficiency and impaired host defence to Mycobacterium tuberculosis. Tuberculosis 1985;66:301-4.

7 Maxwell JD. Seasonal variation in vitamin D. Proc Nutr Soc 1994;53:515-25.

8 Finch PJ, Ang L, Eastwood JB, Maxwell JD. Clinical and histological spectrum of osteomalacia among Asians in south London. $Q \mathcal{F}$ Med 1992;NS 83:439-48.

9 Yang S, Smith C, Prahl JM, Luo X, DeLuca HF. Vitamin D deficiency suppresses cell-mediated immunity in vivo. Arch deficiency suppresses cell-mediated

10 Rook GAW. Role of activated macrophages in the immunopathology of tuberculosis. Br Med Bull 1988;44:611-23. 